\title{
Association of bone mineral density with DXA-determined adipose tissue volume and concentrations of selected hormones in young adult women
}

\author{
Sowińska-Przepiera Elżbieta, Andrysiak-Mamos Elżbieta, Syrenicz Justyna, Syrenicz Anhelli
}

Department of Endocrinology, Metabolic and Internal Diseases, Pomeranian Medical University, Szczecin, Poland sowprzep@pum.edu.pl

\section{Objectives:}

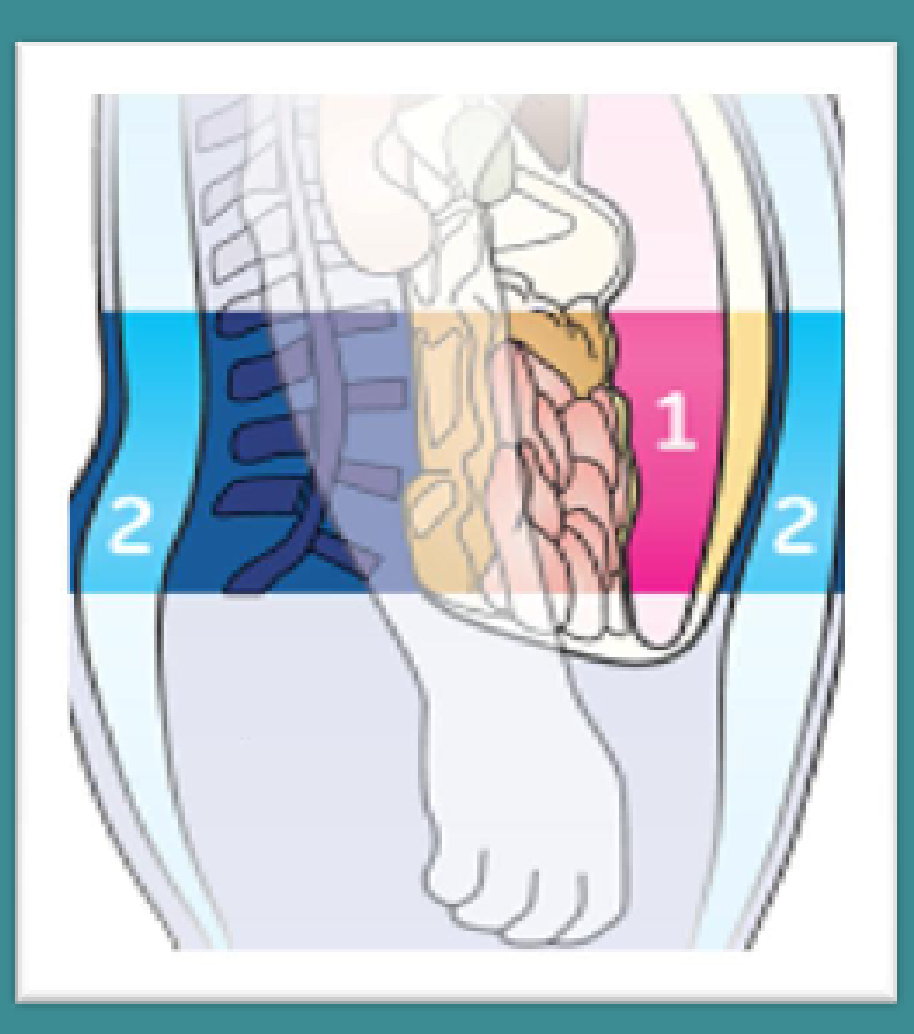

\section{Methods:}
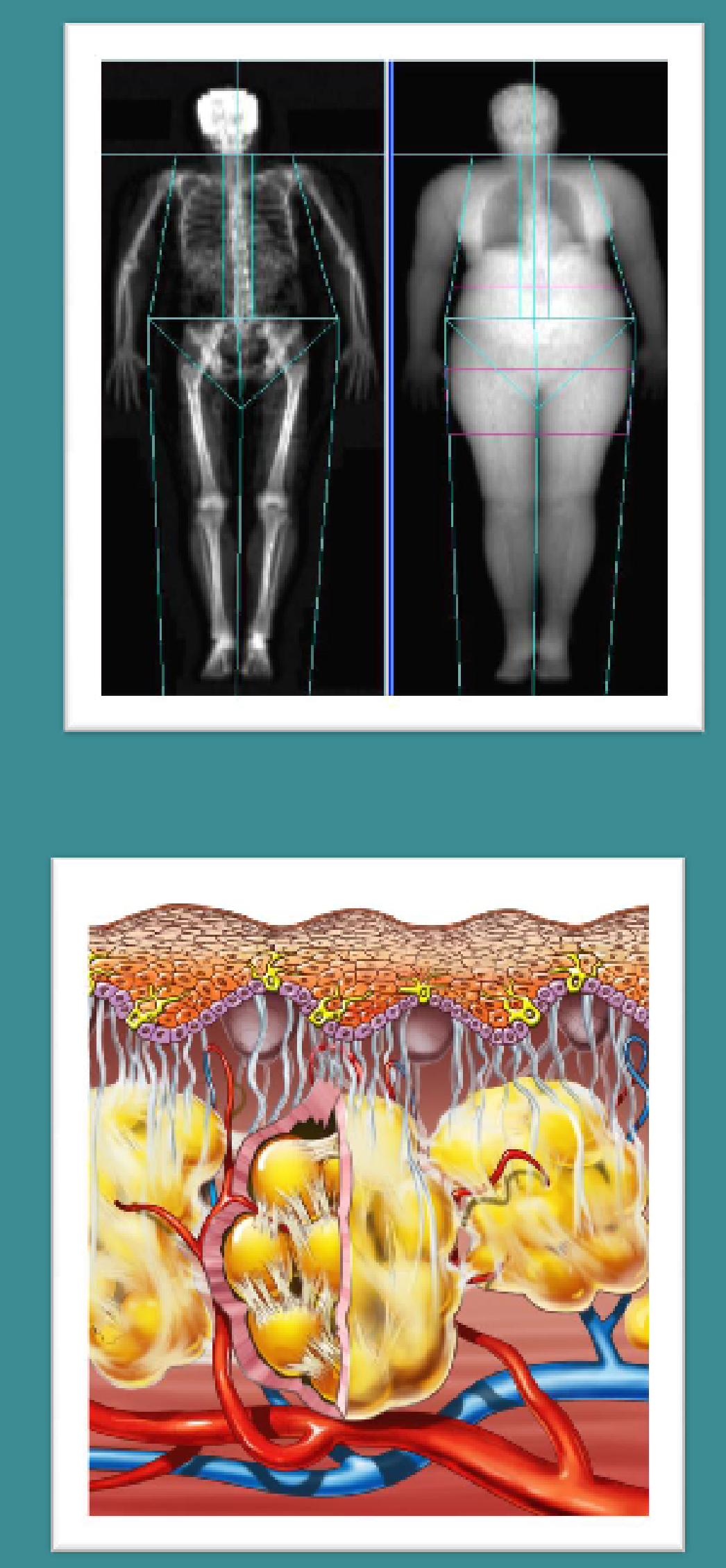

\section{Results:}

\section{Conclusions:}

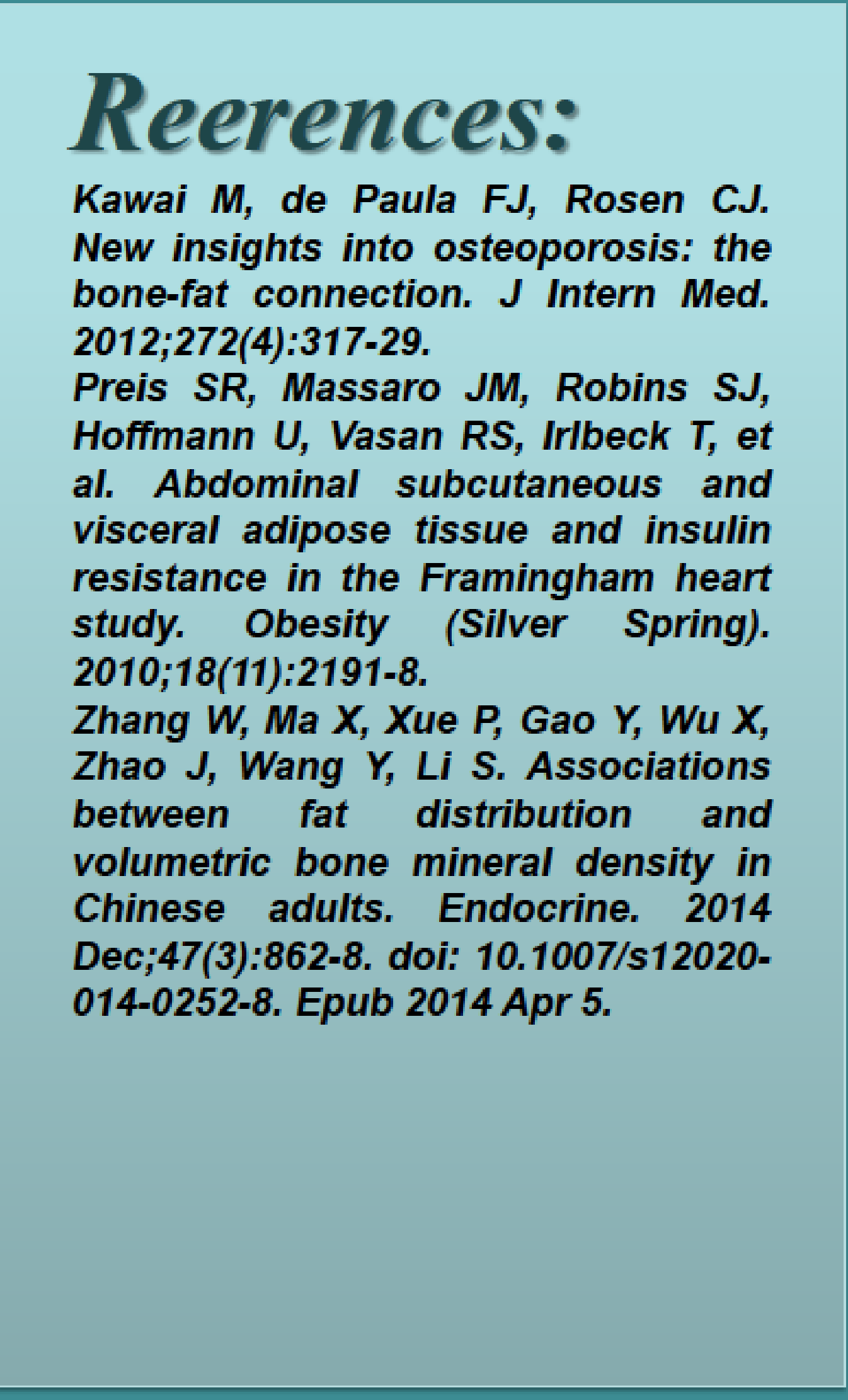

The question if obesity may protect against osteoporosis is still a matter of discussion. A breakthrough in this debate may be the introduction of a state-of-the-art densitometric software (Core Scan) suitable for fast and accurate volumetry of total body fat (BF), including android (A), female-type (F) and visceral fat (VAT) volumes.

The aim of the study was to analyze an association between bone mineral density (BMD]), concentrations of selected hormones, BF, A, F and VAT volumes in young adult women.

Material and methods: The study was a retrospective analysis of densitometric scans and laboratory parameters of 108 women (age: 20-33 years). Two groups of patients were identified based on their body mass index (BMI), with BMI $<25 \mathrm{~kg} / \mathrm{m}^{2}$ and $>25 \mathrm{~kg} / \mathrm{m}^{2}$. The list of analyzed variables included body height and weight, BMI, BMD, DXA-determined DXA (GE Lunar Prodigy; Madison, WI, USA; 14CoreScan TM H8801CP) BF, A, F and VAT volumes, concentrations of TSH, FT3, FT4, FSH, LH, estradiol, PRL, DHEA-SO4, androstendion, testosterone, SHBG, 17-hydroxyprogesterone, levels of glucose and insulin measured after an overnight fast and during glucose tolerance test.

Results: Irrespective of the analyzed area (BF, A, F, VAT), adipose tissue volume correlated significantly with BMD L1-L4, BMD total, blood concentrations of free estrogens and testosterone $(p<0.000)$. Moreover, a significant relationship was found between VAT volume and concentrations of insulin after an overnight fast, as well as at 60 and $120 \mathrm{~min}$ of glucose tolerance test $(p<0.000)$. Finally, VAT volume correlated inversely with blood concentration of SHBG $(p<0.003)$. The two groups of patients differed significantly in terms of their mean values of densitometric parameters $(p<0.000)$.

Greater volume of subcutaneous and visceral fat is likely associated with higher BMD in young adult women and therefore may protect them against osteoporosis. However, high volume of body fat may also predispose to polycystic ovary syndrome and type 2 diabetes mellitus.

\begin{tabular}{|l}
\multicolumn{1}{c}{ Parameter } \\
\hline Age \\
\hline Growth [cm] \\
\hline Body mass [kg] \\
\hline TSH [ug/ml] \\
\hline fT3 [pg/ml] \\
\hline fT4 [ng/ml] \\
\hline FSH [mlU/ml] \\
\hline LH [mlU/ml] \\
\hline Estradiol [pg/ml] \\
\hline Prolactin [ng/ml] \\
\hline DHEAS [ug/ml] \\
\hline Androstendion [ng/ml] \\
\hline Testosterone [ng/ml] \\
\hline SHBG [nmol/l] \\
\hline 17-0HP [ng/ml] \\
\hline BMD L ${ }_{1-4}\left[\mathrm{~g} / \mathrm{cm}{ }^{2}\right]$ \\
\hline T-score \\
\hline BMD total body [cm²] \\
\hline T-score \\
\hline Total body fat (BF) [g] \\
\hline Total body fat (BF) [\%] \\
\hline Including android (A) [g] \\
\hline Including android (A) [\%] \\
\hline female-type (F) [g] \\
\hline female-type (F) [\%] \\
\hline visceral fat (VAT) [g] \\
\hline visceral fat (VAT) [cm 3$]$ \\
\hline P<0,05* \\
\hline
\end{tabular}

\begin{tabular}{cc}
$\begin{array}{c}\text { Group A } \\
\text { BMI }<25 \\
S D\end{array}$ & $\begin{array}{c}\text { Group B } \\
\text { BMI }>25 \\
S D\end{array}$ \\
27,2 & 27,4 \\
\hline 165,4 & 167,3 \\
\hline 58,7 & 88,9 \\
\hline 2,15 & 2,34 \\
\hline 3,07 & 3,18 \\
\hline 1,20 & 1,24 \\
\hline 7,78 & 5,39 \\
\hline 13,9 & 11,10 \\
\hline 68,9 & 74,4 \\
\hline 19,3 & 27,0 \\
\hline 253,5 & 272,5 \\
\hline 3,88 & 4,13 \\
\hline 0,27 & 0,49 \\
\hline 80,26 & $46,49^{*}$ \\
\hline 1,33 & 1,43 \\
\hline 1,15 & $1,26^{*}$ \\
\hline 0,22 & $0,69^{*}$ \\
\hline 1,12 & $1,25^{*}$ \\
\hline 0,36 & $1,66^{*}$ \\
\hline 57068 & 88115 \\
\hline 31,70 & 43,93 \\
\hline 3922 & 6998 \\
\hline 29,8 & 50,5 \\
\hline 9743 & 13944 \\
\hline 35,4 & 43,9 \\
\hline 196 & $1091^{*}$ \\
\hline 208 & $1156^{*}$ \\
\hline & \\
\hline
\end{tabular}

